\title{
Simulating microbial degradation of organic matter in a simple porous system using the 3-D diffusion-based model MOSAIC
}

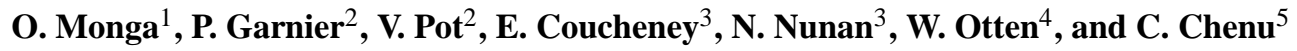 \\ ${ }^{1}$ UMMISCO-Cameroun, Unité Mixte Internationale de recherche UMMISCO, Université de Yaoundé 1 (Cameroun), Institut \\ de Recherche pour le Développement (IRD), Université de Paris 6, Paris, France \\ ${ }^{2}$ INRA, AgroParisTech, UMR1091 EGC, 78850, Thiverval-Grignon, France \\ ${ }^{3}$ CNRS, UMR7618 - Biogéochimie et Ecologie des Milieux Continentaux, 78850, Thiverval-Grignon, France \\ ${ }^{4}$ The SIMBIOS Centre, University of Abertay Dundee, Kydd Building, Dundee, DD1 1HG, UK \\ ${ }^{5}$ AgroParisTech, UMR7618 - Biogéochimie et Ecologie des Milieux Continentaux, 8850, Thiverval-Grignon, France
}

Correspondence to: O. Monga (olivier.monga@ird.fr)

Received: 1 July 2013 - Published in Biogeosciences Discuss.: 2 October 2013

Revised: 7 March 2014 - Accepted: 7 March 2014 - Published: 22 April 2014

\begin{abstract}
This paper deals with the simulation of microbial degradation of organic matter in soil within the pore space at a microscopic scale. Pore space was analysed with microcomputed tomography and described using a sphere network coming from a geometrical modelling algorithm. The biological model was improved regarding previous work in order to include the transformation of dissolved organic compounds and diffusion processes. We tested our model using experimental results of a simple substrate decomposition experiment (fructose) within a simple medium (sand) in the presence of different bacterial strains. Separate incubations were carried out in microcosms using five different bacterial communities at two different water potentials of -10 and $-100 \mathrm{~cm}$ of water. We calibrated the biological parameters by means of experimental data obtained at high water content, and we tested the model without changing any parameters at low water content. Same as for the experimental data, our simulation results showed that the decrease in water content caused a decrease of mineralization rate. The model was able to simulate the decrease of connectivity between substrate and microorganism due the decrease of water content.
\end{abstract}

\section{Introduction}

It is increasingly recognized that accessibility is one of the major factors governing soil organic matter decomposition (Dungait et al., 2012). Indeed, soil microorganisms live in a complex network of pores, resulting from the threedimensional arrangement of soil solid particles. This network is more or less filled with air and water, variously interconnected and in which microorganisms as well as organic resources are spatially heterogeneous (Chenu and Stotzky, 2002; Nunan et al., 2003, 2007; Young et al., 2008). Microbial degradation of soil organic matter is then expected to depend on the accessibility of organic matter to microorganisms at the microscale (i.e. on their spatial co-occurrence or separation, and on the rates of diffusion of substrates and enzymes, as well as on local favourable conditions for the microorganisms).

Current models of soil organic matter dynamics, such as CENTURY (Kelly et al.,1997) and RothC (Coleman et al., 1997), do not take such microscale processes into account, but they typically are process-oriented multi-compartmental models that divide soil organic matter into conceptual pools with distinct turnover times, assuming that a combination of biochemical and physical properties controls decay (Manzoni and Porporato, 2009). Soil texture or clay content is used in some models to modify decomposition processes, but the majority of the models treat soil as homogeneous. These models are capable of simulating soil organic matter dynamics at long timescales (Smith et al., 1997), but they are limited in their ability to predict short-term changes in soil organic matter (SOM) degradation or to account for changes in soil structure or moisture (Gottschalk et al., 2010; Falloon et al., 2011). Mechanistic representation of small-scale processes is 
identified as one of the priorities to improve soil organic matter dynamics models (Manzoni and Porporato, 2009). Recent modelling efforts have attempted to understand how microbial processes such as decomposition or competition among species are affected by diffusion in 1-D or 2-D homogeneous porous media (Long and Or, 2009; Ingwersen et al., 2008). A few recent studies have also simulated microbial degradation in structured environments. Gharasoo et al. (2012) have developed a 2-D pore network model able to simulate the biodegradation of a dissolved contaminant in a virtual pore network according to different scenarios of microbial spatial distributions. Resat et al. (2012) have built a model where soluble substrate and enzyme kinetics were described with continuous partial differential equations on a 3-D grid. The pore distribution in aggregates was projected onto a constructed lattice grid where each grid unit was labelled with a pore parameter, which defined it as solid or porous.

So far the spatial complexity of the pore network in real soils has rarely been explicitly represented. Exceptions include recent work by Kravchenko et al. (2012), who used a 3D microscopic-based biophysical model to explore how management affects fungal colonization and interaction. This is partly because it has been possible only recently to visualize the soil pore network, using X-ray microtomography (Peth et al., 2008; Mooney, 2002; Wildenschild and Sheppard, 2013) and therefore obtain the data necessary to produce an explicit description of the microscale structure of soil. Computed tomography images provide a first rough pore space representation by means of a set of voxels. However, the size of this representation, typically up to 30 million of voxels, is too high to be used effectively for simulating soil processes in the pore space, and is restricted to relatively small volumes of soil (Kravchenko et al., 2011). Using X-ray tomography images as model input data, Monga et al. (2007) derived from this a network of volume primitives to produce a geometrical representation of pore space in soil that had similar properties (i.e. total pore volume, pore connectivity) to those of real soil samples. Biological activity was incorporated and the decomposition of organic matter was simulated at the microscale (Monga et al., 2008). The latter model did not, however, take into account the diffusion of dissolved organic matter that we believe to be an important regulator of microbial decomposition in soil.

The objective of the present paper is to test our modelling approach, partly described in Monga et al. $(2008,2009)$ with real data of soil structure and measurements of decomposition of dissolved organic matter. We introduced the diffusion process into the graph-based approach using Fick's laws to simulate mass exchanges between pores. We used an experimental system where it was possible to control and measure variables that enabled us to test and parameterize our model (soil pore space geometry together with biological variables). Specifically, we experimentally quantified the effect of moisture content within a soil structure on the microbial degradation of fructose. We investigated the performance of the model by testing its ability to reproduce (i) the water retention relationship for the microcosms and (ii) the consequence of water distribution on the microorganisms respiration. We compared the results of our 3-D modelling approach with those obtained using a moisture limiting function from the literature.

\section{Material and methods}

\subsection{MOSAIC II model}

As described in Monga et al. (2007) and Ngom et al. (2012), we approximated the geometry of the pore space by a network of volume primitives. To do so, we used a geometrical algorithm based on Delaunay triangulation to calculate the set of maximal spheres that describe the pore space geometry. Then we extracted a minimal set of the maximal spheres in order to obtain a compact representation of the pore space. A relational attributed valuated graph ("graph-based approach") was finally attached to the spheres (Monga et al., 2007). Let $G(t)=\left(B_{i}, A_{i}, F_{i}(t)\right)$ be the valuated graph, where $\left(B_{i}\right)$ denotes the set of nodes (spheres) of the graph, $\left(A_{i}\right)$ the set of arcs and $\left(F_{i}(t)\right)$ the feature vector defining the physical and biological parameters of the nodes $i$ at a given time $t$. We assumed that the pore space does not vary, and therefore only the biological features and dissolved organic matter (DOM) depend on time as detailed below.

In previous version of our model (Monga et al., 2008), we modelled organic matter decomposition using an offer-demand approach (Masse et al., 2007). We assumed that, depending on the minimum values of the degradation rate of solid organic matter or microbial growth rate (both expressed in $\mathrm{C}$ units), one of these two kinetics was dominating the decomposition rate. Here we extended the model by including DOM, coming from the hydrolysis of solid organic matter as one intermediate compartment that is often included in soil organic matter models (e.g. Garnier et al., 2003). A geodesic distance was previously used to connect DOM and microbial biomass located in different pores. The geodesic distance led to an immediate availability of the substrate for microorganisms if connecting paths exist. In this new version of the model, we modified the organic matter decomposition module in order to describe the production of dissolved organic matter by hydrolysis of solid organic matter and introduce mechanistic diffusion of DOM in pores. As in soils, DOM diffuses between microbial habitats, where it is assimilated and mineralized. The microbial decomposition simulation was processed by graph updating using time discretization as described in Monga et al. (2008).

Let $B_{k}$ in $G$ be a node attached to a volume primitive (i.e. pore). The biological and physical features $F_{k}(t)$ that describe $B_{k}$ are as follows: 


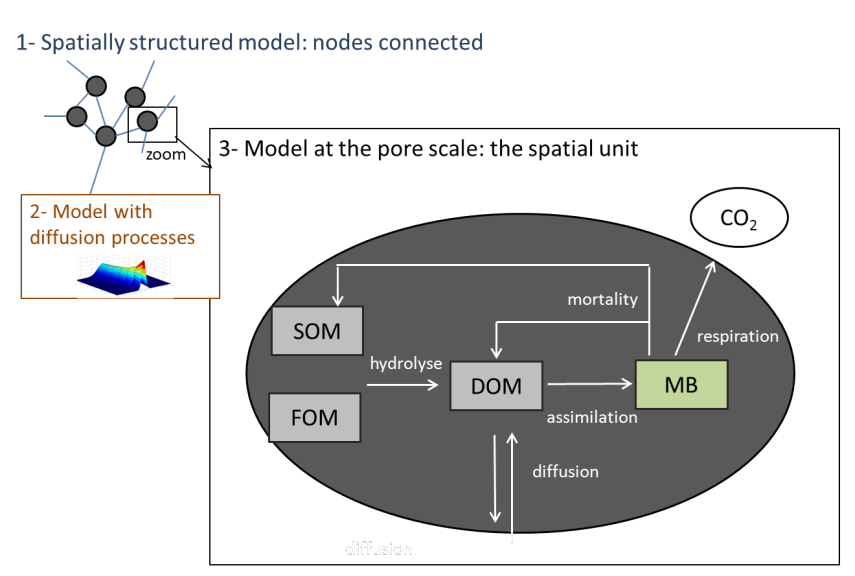

Fig. 1. Biological model.

- $F_{k}(1)(t)$ : mass of microbial biomass (MB) (gC),

- $F_{k}(2)(t)$ : mass of dissolved organic matter (DOM) $(\mathrm{gC})$,

- $F_{k}(3)(t)$ : mass of soil organic matter (SOM) $(\mathrm{gC})$,

- $F_{k}(4)(t)$ : mass of fresh organic matter (FOM) $(\mathrm{gC})$,

- $F_{k}(5)(t)$ : mass of inorganic carbon $\mathrm{CO}_{2}(\mathrm{gC})$,

- $F_{k}(6)(t)$ : presence of water or air,

- $F_{k}(7)(t)$ : volume of the sphere.

We described the microbial decomposition process with five compartments (Fig. 1), namely MB (microbial biomass), FOM (fresh organic matter), SOM (soil organic matter), DOM (dissolved organic matter) and $\mathrm{CO}_{2}$ (mineralized organic matter). FOM and SOM are decomposed rapidly and slowly, respectively. DOM comes from the hydrolysis of SOM and FOM. DOM diffuses through water paths (waterfilled spheres) and is consumed by MB for its growth. We hypothesized that MB does not move. Dead microorganisms are recycled into SOM and DOM. MB respires by producing inorganic carbon $\left(\mathrm{CO}_{2}\right)$, as represented in Fig. 1 .

The changes of the biological features $F_{i}(t)$ within a time step $\Delta t$ in a water-filled sphere $B_{i}$ are expressed as follows:

$$
\begin{aligned}
& F_{i}(1)(t+\Delta t)=F_{i}(1)(t)-\rho F_{i}(1)(t) \\
& \Delta t-\mu F_{i}(1)(t) \Delta t+\left(\frac{\vartheta_{\mathrm{DOM}} F_{i}(2)(t)}{K_{b} \times F_{i}(7)(t)+F_{i}(2)(t)}\right)
\end{aligned}
$$

$F_{i}(1)(t) \Delta t$,

$$
\begin{aligned}
& F_{i}(2)(t+\Delta t)=F_{i}(2)(t)+p_{m} \mu F_{i}(1)(t) \\
& \Delta t-\left(\frac{\vartheta_{\mathrm{DOM}} F_{i}(2)(t)}{K_{b} \times F_{i}(6)(t)+F_{i}(2)(t)}\right) F_{i}(1)(t) \\
& \Delta t+\vartheta_{\mathrm{SOM}} F_{i}(3)(t) \Delta t+\vartheta_{\mathrm{FOM}} F_{i}(4)(t) \Delta t,
\end{aligned}
$$

$$
\begin{aligned}
& F_{i}(3)(t+\Delta t)=F_{i}(3)(t)+\left(1-p_{m}\right) \mu F_{i}(1)(t) \\
& \Delta t-\vartheta_{\mathrm{SOM}} F_{i}(3)(t) \Delta t, \\
& F_{i}(4)(t+\Delta t)=F_{i}(4)(t)-\vartheta_{\mathrm{FOM}} F_{i}(4)(t) \Delta t, \\
& F_{i}(5)(t+\Delta t)=F_{i}(5)(t)+\rho F_{i}(1)(t) \Delta t,
\end{aligned}
$$

where $\rho$ is the relative respiration rate $\left(\mathrm{d}^{-1}\right), \mu$ the relative mortality rate $\left(\mathrm{d}^{-1}\right), p_{m}$ the proportion of MB that returns to DOM (the other fraction returns to SOM), $\vartheta_{\text {FOM }}$ and $\vartheta_{\text {SOM }}$ the relative decomposition rates of FOM and SOM respectively $\left(\mathrm{d}^{-1}\right)$, and $\vartheta_{\text {DOM }}\left(\mathrm{d}^{-1}\right)$ and $K_{b}(\mathrm{gC})$ the maximum relative growth rate of $\mathrm{MB}$ and constant of half-saturation of DOM by MB, respectively.

In MOSAIC II, the implementation of the diffusion process of DOM within water-filled pore space by updating the valuated graph representing the pore network was performed according to classical diffusion scheme. Diffusion of DOM between two connected water-filled pores $\left(B_{k}, B_{p}\right)$ can be expressed as the material exchange $\delta m_{k p}$ :

$\delta m_{k p}=\frac{-D\left(d_{k}-d_{p}\right) S_{k p} \delta t}{g_{k p}}$

with

$$
\begin{aligned}
& d_{k}=\frac{F_{k}(2)(t)}{\frac{4}{3} \pi r_{k}^{3}} \\
& d_{p}=\frac{F_{p}(2)(t)}{\frac{4}{3} \pi r_{p}^{3}},
\end{aligned}
$$

where $D$ denotes the molecular diffusion coefficient of DOM in water $\left(\mathrm{cm}^{2} \mathrm{~d}^{-1}\right), g_{k p}$ corresponds to the distance between the two water-filled pores $(\mathrm{cm})$ (calculated as the distance between the centres of two neighbouring water-filled spheres), $d_{k}$ and $d_{p}$ denote the DOM concentration within the waterfilled pores $B_{k}$ and $B_{p}\left(\mathrm{gC} \mathrm{cm}^{-3}\right)$, respectively, and $r_{k}$ and $r_{p}$ denote the radius of the pores $B_{k}$ and $B_{p}(\mathrm{~cm}) . S_{k p}$ is the contact surface between the two water-filled pores $\left(\mathrm{cm}^{2}\right)$.

The DOM mass variation due to diffusion can then be expressed as follows (first Fick law):

$F_{k}(2)(t+\Delta t)=F_{k}(2)(t)+\delta m_{k p}$,
$F_{p}(2)(t+\Delta t)=F_{p}(2)(t)+\delta m_{k p}$.

The mass transfer between water-filled pores $B_{k}$ and $B_{p}$ between time $t$ and $t+\mathrm{d} t$ is calculated at $t$. If the total estimated mass transfer for pore $B_{k}, \Delta_{m k}$ exceeds $F_{k}(2)(t)$, then the available mass is distributed proportionally to the neighbouring pores.

Equations (1)-(10) express the change of the biological and biochemical vectors describing each of the water-filled pores (graph node) within time step $\Delta t$. These equations correspond to the discrete version of a partial differential equation system (in 3-D space) and describe the biological model MOSAIC II. 
The distribution of air and water in the sphere network was performed by applying an algorithm based on the YoungLaplace law in the sample border to determine spheres filled with water according to water potential (Monga et al., 2008):

$\Delta p=\frac{2 \sigma \cos \theta}{r_{\mathrm{eq}}}$,

with $\Delta p$ the capillary pressure $(\mathrm{m}), r_{\mathrm{eq}}$ the equivalent maximum radius of water-filled pores (m) at $\Delta p, \alpha g$ the contact angle equal to 0 , and $\sigma$ the surface tension of water equal to $0.0728 \mathrm{~N} \mathrm{~m}^{-1}$. According to the capillary pressure, the spheres $B_{i}$ were either filled by water $\left(F_{i}(6)(t)=1\right)$ or empty (full of air, $F_{i}(6)=0$ ). Then an iterative drainage algorithm was used to drain the remaining water inside the sample if it was connected to air pathway (Monga et al., 2008). A water retention curve was predicted this way by calculating the total volumetric water content as a function of pressure.

\subsection{Biodegradation experiment}

In order to test the model, we performed experiments in which we monitored the mineralization of a simple soluble substrate, fructose, by different single bacterial species in sand microcosms. Sand was selected as a simplified variant of soil architecture and because it does not add additional SOM to the system. The 3-D structure of soil pore space of the sand was obtained by X-ray microCT (described below).

A range of bacterial species with differing physiological properties were selected: three Arthrobacter species renamed as 3R, 7R, and 9R; and two Rhodococcus species renamed as $5 \mathrm{~L}$ and $6 \mathrm{~L}$. The different bacterial strains tested were isolated from a soil and selected for their ability to grow in a minimal medium amended with fructose in aerobic conditions (Coucheney, 2009). Microcosms consisted in $60 \mathrm{~g}$ of dry sand, placed in an autoclavable glass jar. Quartz sand of Fontainebleau (Prolabo), with 150-300 $\mu \mathrm{m}$ grain size, was used after rinsing with deionized water and drying in an oven for $1 \mathrm{~h}$ at $500^{\circ} \mathrm{C}$ in order to oxidize any remaining organic matter present. An amount of pure bacterial cultures (Approx. $10^{\wedge 6}$ cells) of each species was inoculated in the sterile sand microcosms with a minimal medium $\left(1.28 \% \mathrm{Na}_{2} \mathrm{HPO}_{4}-7 \mathrm{H}_{2} \mathrm{O}, 0.3 \% \mathrm{KH}_{2} \mathrm{PO}_{4}, 0.05 \%\right.$ $\mathrm{NaCl}, 0.1 \% \mathrm{NH}_{4} \mathrm{Cl}, 0.024 \% \mathrm{MgSO}_{4}$ and $0.001 \% \mathrm{CaCl}_{2}$; Sambrook et al., 1989) containing fructose as a sole carbon source ( $8 \mathrm{mg} \mathrm{C} / \mathrm{microcosm}$ i.e. $0.13 \mathrm{mg} \mathrm{g}^{-1}$ sand) in three replicates. The minimal medium containing the bacterial suspension was thoroughly mixed with the sand under sterile conditions, disposed in a Petri dish and compacted at a fixed density of $1.56 \mathrm{~g} \mathrm{~cm}^{-3}$. The amount of water was adjusted in order to achieve two contrasting gravimetric water contents of 0.216 and $0.087 \mathrm{~g} \mathrm{H}_{2} \mathrm{O} \mathrm{g}^{-1}$ sand (equivalent to water potentials of -10 and $-100 \mathrm{~cm}$, respectively). The concentrations of fructose were 0.26 and $1.53 \mathrm{mg} \mathrm{C} \mathrm{cm}^{-3}$ of water for high and low water contents, respectively. The concentrations of bacteria were $4.10^{-9}$ and $10^{-8} \mathrm{mg} \mathrm{C} \mathrm{cm}^{-3}$ of water for high and low water contents, respectively (we assumed that one bacterium contains $5 \cdot 10^{-14} \mathrm{mg} \mathrm{C}$ ). Each microcosm was then closed inside an air-tight jar and incubated for a week at $25^{\circ} \mathrm{C}$. An aliquot of a few microlitres of the atmosphere was taken daily and analysed by gas chromatography (Agilent 3000A) in order to measure $\mathrm{CO}_{2}$ concentration in the atmosphere of the jar. All measurements were replicated three times.

\subsection{Physical characterization of the sand}

The water release curve of the sand was measured using pressure plates $(n=3)$. The total porosity of the sand column calculated from the water retention curve was $38 \%$. The sand column was scanned with a high-resolution X-ray microcomputed tomography machine ( $\mu$ SIMCT Equipment: SIMBIOS Centre University of Abertay Dundee, Scotland) at a voxel resolution of $5 \mu \mathrm{m}$. For the numerical simulations, we extracted an image of $500 \times 500 \times 500$ voxels (i.e. $15 \mathrm{~mm}^{3}$ ) from the total 3-D image, which was the largest sample we could comfortably cope with given the memory requirements and computing times. For the simulations we have done, on a regular PC, a few hours (2-3 h) were necessary to run one simulation with diffusion. A global threshold was used to segment the CT images and identify the pore space within the 3-D samples. The threshold value was adjusted in order to fit a porosity of $31 \%$. This was the porosity calculated from the experimental water retention curve considering pores with diameters larger than $5 \mu \mathrm{m}$ (i.e. pores filled with water between -600 and $0 \mathrm{~cm}$ of water).

\subsection{Simulating microbial decomposition by graph updating with Mosaic II}

As fructose is a soluble molecule and the sand was OM-free, we assumed that initially only MB (microbial biomass) and DOM (dissolved organic matter) were present within pores. The DOM was initially spread in a homogeneous way within the water-filled pores space with the same amount but with different concentrations for each water content. The MB was initially spread randomly within the water-filled pore space also with the same amount but with higher concentration for lower water content. At the start of the simulation, SOM and $\mathrm{CO}_{2}$ were equal to zero. The duration of the microbial decomposition simulation was 7 days. We assumed that oxygen was not a limiting factor of biodegradation.

We emptied the spheres of their water according to the drainage algorithm. For this, we calculated the radius thresholds, $r_{1}$ and $r_{2}$, given by Young-Laplace law (Eq. 12) for the two water potentials of the experiment, which were $\Delta p_{1}=$ $-10 \mathrm{~cm}$ (high water potential) and $\Delta p_{2}=-100 \mathrm{~cm}$ (low water potential) as being respectively $145 \mu \mathrm{m}$ and $14.5 \mu \mathrm{m}$. In the following components of the simulation, we retained only the water-filled pores. 
Table 1. Parameter values estimated by the calibration of MOSAIC II from carbon mineralization curves registered from incubation of each species with fructose at high water content (where $\vartheta_{\mathrm{DOM}}$ is the maximum growth rate, $K_{b}$ is the constant of half-saturation, $\mu$ is the mortality, $\rho$ is the respiration, $p_{m}$ is the proportion of MB that returns to DOM) with their respective efficiency coefficients.

\begin{tabular}{lllllll}
\hline Species & \multicolumn{7}{c}{ Parameters } \\
\cline { 2 - 7 } & $\begin{array}{l}\vartheta_{\mathrm{DOM}} \\
\left(\mathrm{day}^{-1}\right)\end{array}$ & $\begin{array}{l}K_{b} \\
\left(\mathrm{gCg}^{-1}\right)\end{array}$ & $\begin{array}{l}\mu \\
\left(\text { day }^{-1}\right)\end{array}$ & $\begin{array}{l}\rho \\
\left(\text { day }^{-1}\right)\end{array}$ & $\begin{array}{l}p_{m} \\
(\%)\end{array}$ & EF \\
\hline Arthrobacter sp. 3R & 17 & 0.0005 & 1.5 & 0.2 & 70 & 0.84 \\
Arthrobacter sp. 9R & 9.6 & 0.001 & 0.5 & 0.2 & 55 & 0.91 \\
Arthrobacter sp. 7R & 8 & 0.00014 & 1 & 0.3 & 20 & 0.98 \\
Rhodococcus sp. 6L & 9 & 0.0005 & 0.22 & 0.45 & 20 & 0.8 \\
Rhodococcus sp. 5L & 8.16 & 0.0007 & 0.4 & 0.25 & 55 & 0.94 \\
\hline
\end{tabular}

Simulations were carried out in two steps. In the first step, the five parameters of the model $\left(\vartheta_{\mathrm{DOM}}, K_{b}, \mu, \rho, p_{m}\right)$ were calibrated using the experimental $\mathrm{CO}_{2}$ mineralization curve obtained at the high water potential of $-10 \mathrm{~cm}$ (Table 1) for each species. We used the same diffusion parameter for all species. The parameter estimation was carried out by trial and error.

In the second step, we tested the model with the data obtained at low water potential of $-100 \mathrm{~cm}$. We kept the same biological parameters obtained at the high water potential. We assumed that the differences of mineralization between both water contents came from the diffusion limitation due to the lower soil moisture. We hypothesized that the physiology of microorganisms (i.e. their maximal growth rate, constant of half saturation or mortality rate) was not affected by the decrease in moisture, because the water potential remained too high to cause a physiological stress (Manzoni et al., 2012). The bacteria are supposed to be attached to the solid particles and are submitted to the same micro-environment at -10 or $-100 \mathrm{~cm}$ of water. Several studies have found that diffusion limitation was the main factor explaining the decrease of respiration with soil moisture (e.g. Harms, 1996; Dechesne et al., 2010; Moyano et al., 2013). A meta-analysis by Manzoni et al. (2012) showed that moisture soil respiration curves were not affected by microbial community composition, which they interpreted by other factors than microbial physiology controlling respiration (i.e. solute diffusivity).

\subsection{Simulating microbial decomposition with a simple approach}

We compared also our results with the results obtained from a simpler approach. We simulated the effect of moisture on degradation by using a simple moisture limitation function instead of calculating the change in connected water pathway with Mosaic II. The biological system of Fig. 1 was simulated in one sphere of Mosaic II to remove the spatial dimension. Parameters of step 1 were used to simulate the lower water potential of $-10 \mathrm{~cm}$. We simulated the water potential of $-100 \mathrm{~cm}$ by using the limitation function used in
RothC and described in Jenkinson and Coleman (2008). The microbial growth rate $(\mu)$ was multiplied by this limitation function equal to 0.7 for the water content change from -10 to $-100 \mathrm{~cm}$ (Fig. 5).

\subsection{Statistics}

The effectiveness of simulations was determined by calculating the efficiency coefficient (EF) for $\mathrm{CO}_{2}$ mineralization curve of each bacterial species:

$\mathrm{EF}=\frac{\sum_{i=1}^{n}\left(O_{i}-\bar{O}\right)^{2}-\sum_{i=1}^{n}\left(S_{i}-\bar{O}\right)^{2}}{\sum_{i=1}^{n}\left(O_{i}-\bar{O}\right)^{2}}$,

where $n$ is the number of experimental observations of $\mathrm{CO}_{2}$; $S_{i}$ and $O_{i}$ are the simulated and observed values, respectively; and $O$ is the average of $n$ experimental values.

\section{Results and discussion}

\subsection{Simulation of sand structure and water retention}

Figure 2 shows 2-D sections of the CT images of the sand microcosms and the segmented images (Fig. 2c) that were used as the initial data to define the pore space and parameterize the physical model. Figures 3 and 4 present the resulting minimal set of maximal spheres of the sand pore space. We applied the drainage algorithm for the two radius thresholds, and we obtained different sphere networks depending on water potential, which included 860710 and 782691 water-filled spheres for the high and low water potentials respectively with a percentage of water in total pore volume of $99 \%$ and $24 \%$, respectively. We found 704 and 43067 sets of connected spheres for high and low water potentials, respectively, that occupied an average volume of 80550 and 334 voxels, respectively. These results indicated that although the numbers of water-filled spheres were not so different between both water potentials, the connectivity was strongly affected by the decrease of water content. 


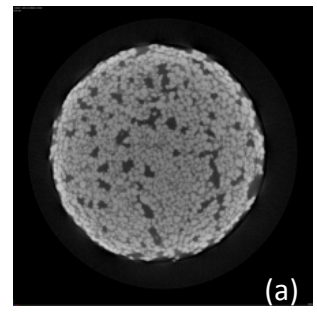

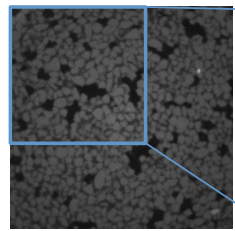

(b)

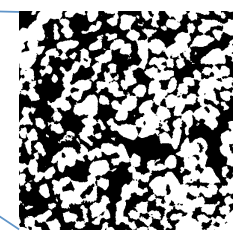

(c)
Fig. 2. View of a slice $(1650 \times 1650$ pixels $)$ of the CT image of the sand (a). View of a slice $(400 \times 400$ pixels $)$ extracted of the $3-\mathrm{D}$ image (b) and segmented image (c). In (a) and (b) the grey level intensity is proportional to the density of the material, while in the segmented image (c) the pore space is in black and the solid phase is in white. The porosity comprising pores $\geq 5 \mu \mathrm{m}$ diameter is $31 \%$.
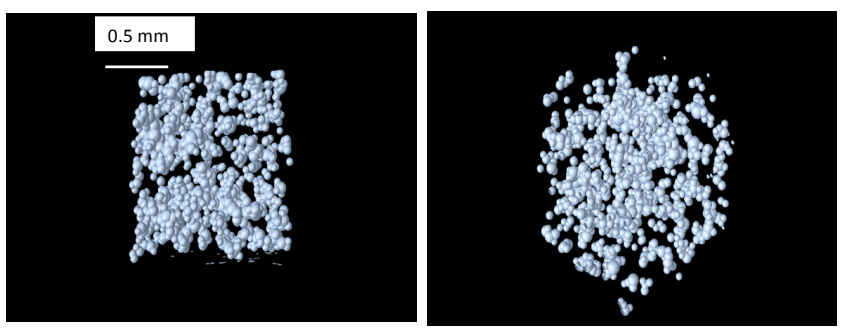

Fig. 3. Perspective views of the sphere-based pore space of the sand. The spheres whose radii were higher than $10 \mu \mathrm{m}$ were displayed.

Figure 5 shows the comparison of the measured water retention curve of sand microcosms and the simulated water distribution using the drainage algorithm. Curves are very close except for pressures near zero where the model overestimated the water content compared to experimental data. In Ngom et al. (2012), we showed that for large pores our model calculated large spheres whose radius is close to the real radius of pores as well as smaller spheres located between the pore wall and the large sphere. According to Young-Laplace law, these smaller spheres remain water-saturated although the equivalent pores, where they are located, should be full of air. This result leads to an overestimation of water content using our geometrical approach at these water pressures near saturation.

\subsection{Simulation of fructose mineralization in sand at two matric potentials}

All bacteria rapidly mineralized fructose, and the experimental results showed, for most bacteria, a slightly higher mineralization at the high water content compared to the one at the lower water content (Fig. 6, day 2). At low water potentials, bacteria are very unlikely affected by a direct physiological effect of the matric potential. Direct adverse effects on bacteria have been shown to occur at lower matric potentials (Holden, 1997; Roberson and Firestone, 1992; Dechesne et al., 2010). It is more likely that the matric poten-

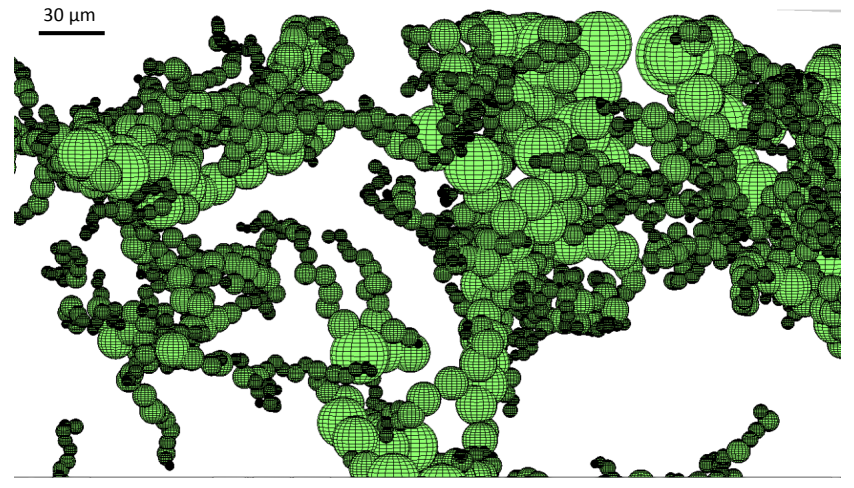

Fig. 4. Details of the maximal spheres covering the pore space of the sand.

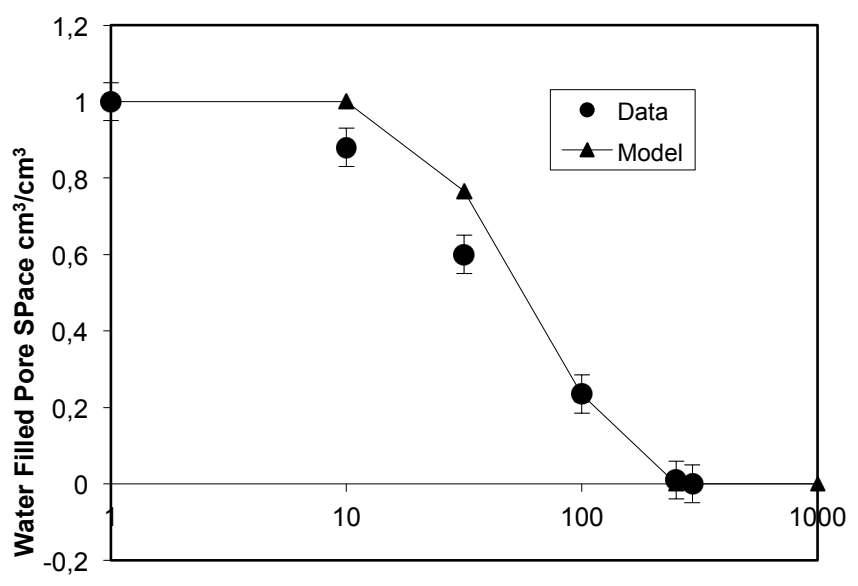

Water pressure head $(-\mathrm{cm})$

Fig. 5. Water retention curves of the sand obtained experimentally and with MOSAIC II.

tial primarily affected mineralization through its control on the substrate diffusion rate through water-filled pores as discussed by Moyano et al. (2013). When the water potential and the water content are smaller, a poorer connection of the water-filled pores is expected. Indeed, the water-filled pores are the diffusion pathways for fructose towards the immobile bacteria. Overall diffusion was presumably limited for the lower water content because of reduced interaction between bacteria and substrates.

Figure 7 shows the bacterial respiration simulated from the best estimated parameter set, the associated measured data performed at the high water content for bacteria $7 R$, and the dynamic for each of the carbon pools of the model. The model describes a rapid decrease of DOM, fructose being consumed within 3 days by the bacteria, a rapid increase of the bacterial biomass and from 2 days its decrease due to mortality. Accordingly, the amount of SOM progressively increases, being fed by $\mathrm{C}$ from dead bacteria. This demonstrates the importance of the different pools described by the 

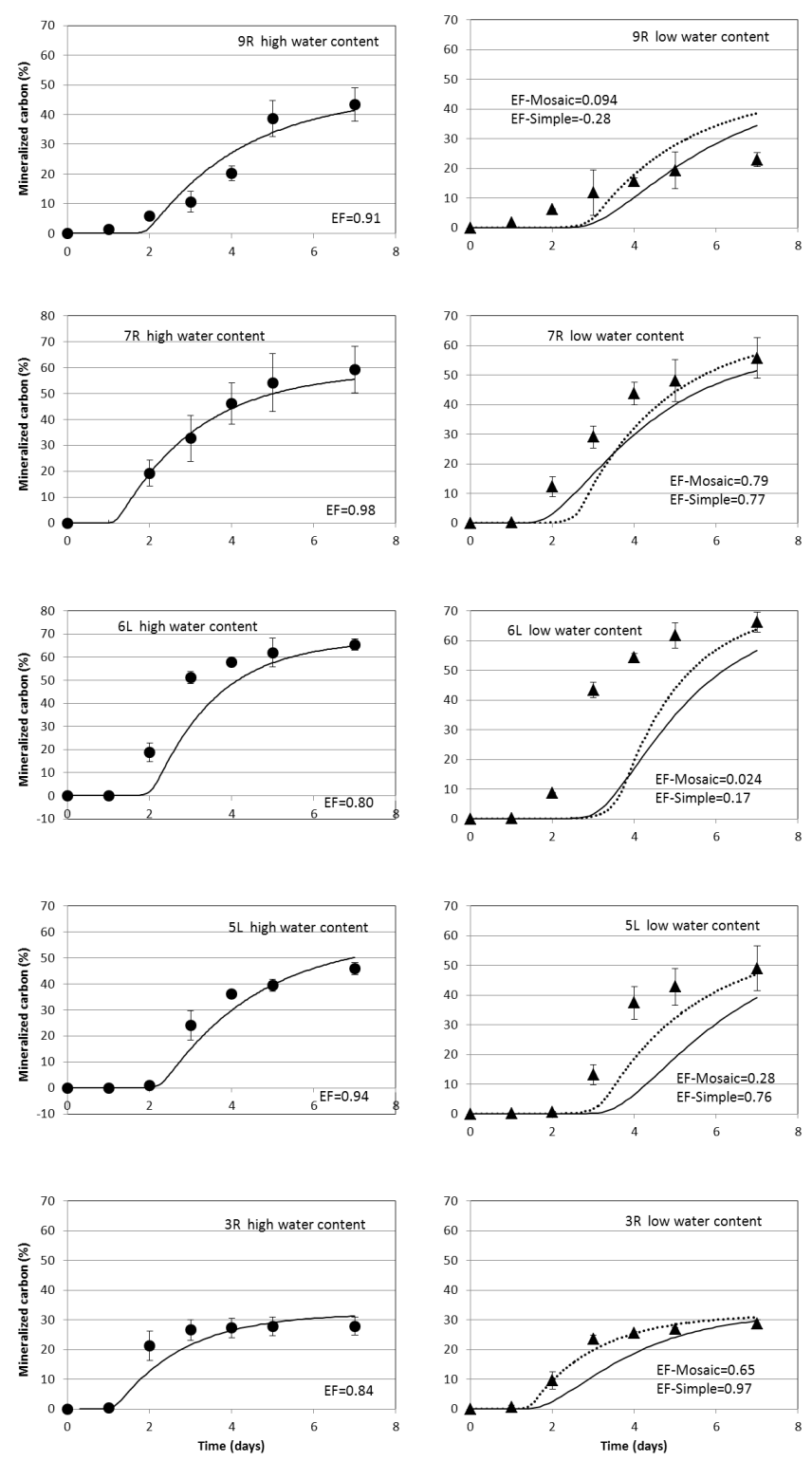

Fig. 6. Experimental (symbols) and simulated (line) results for the five bacterial species inoculated at high and low water contents with their respective efficiency coefficients. For the low water content, simulations with 3-D Mosaic II and with a simpler approach using the moisture function of RothC model are displayed.

model. The shape of the mineralization curve obtained from the calibration at the high water potential was quite similar to the experimental ones, and the total amount of $\mathrm{CO}_{2}$ respired was close to the experimental results. The efficiency coefficient between the simulated mineralized $\mathrm{CO}_{2}$ curve and the measured one was 0.98 . The calibrated parameters - i.e. maximum growth rate, constant of half saturation, mortality and respiration rate for each microbial species - are given in Table 1 . The maximal growth rate $\left(\vartheta_{\text {DOM }}\right)$ estimated between 8 and 17 day $^{-1}$ was of the same order of magnitude as

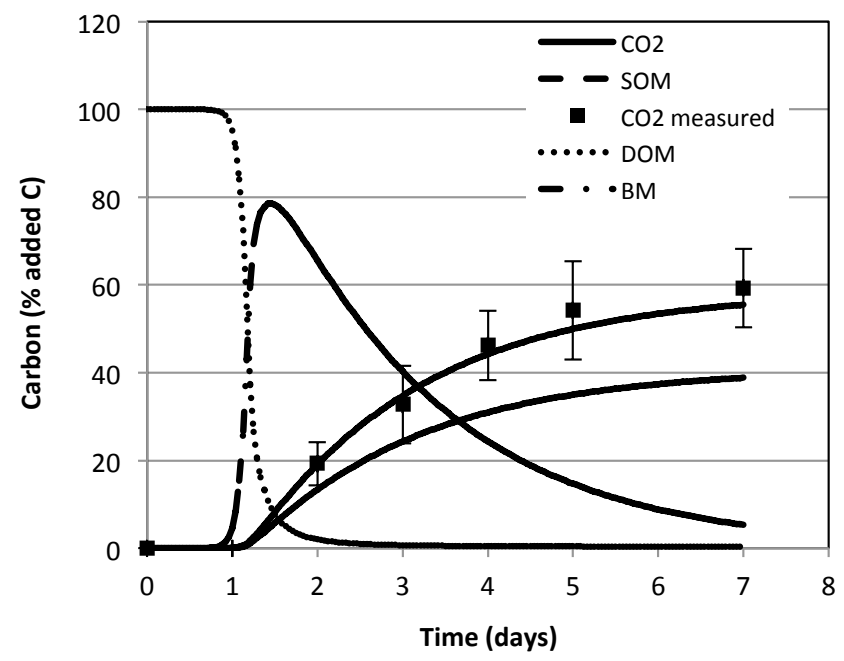

Fig. 7. Calibration of the model parameters from the $\mathrm{CO}_{2}$ measurements during the incubation at high water content, example of bacteria $7 \mathrm{R}$.

those found in soil samples by Ingwersen et al. (2008) and by Treves et al. (2003) (between 1.7 and $44 \mathrm{day}^{-1}$ ). The mortality rate $(\mu)$ was estimated between 0.22 and $1.5 \mathrm{day}^{-1}$. This range is a little bit lower than the one found in soils by Blagodatsky et al. (1998) (between 1.2 and 3.8 day $^{-1}$ ). The respiration rate $(\rho)$ estimated between 0.2 and 0.45 day $^{-1}$ was the same order of magnitude as those used by Gignoux et al. (2001) (between 0.1 and $0.5 \mathrm{day}^{-1}$ ). Most soil organic matter decomposition models use assimilation yields, and consequently very few respiration rate parameters are available in the literature for comparison with our results.

We compared the experimental carbon mineralization curves with the ones calculated by simulation for each bacterial species for the lower water content (Fig. 6). As in the experimental data, we found a lower mineralization for the lower water content. The decrease of water potential led to a lower water content and to another distribution of water among spheres by the model. This new setting changed the pathways of diffusion from fructose to microbial habitats by extending their length and decreasing their number. We found systematic lower efficiency coefficients for the low water content compared to the high water content where parameter estimation had been done. The differences between mineralization curves for high and low water contents were also larger in the simulation than in the experiment (i.e. the effects of water potential were more drastic). We found generally a longer delay for the starting point of the simulated respiration curve compared to the experimental data, especially for the lower water content.

These discrepancies may come from an underestimation of diffusion in the simulation. For example, we do not allow for water films to exist in the simulations but have pores either filled with water or drained. In soils, such water films can act 
as diffusion pathways and connect sites that appear separate otherwise in the simulation. In addition, because of the resolution of $5 \mu \mathrm{m}$ of the microCT scans, the pores of smaller diameter cannot be visualized and hence are not accounted for in the simulation with MOSAIC II. However, such pores may contribute significantly to diffusion of the substrate, especially when the water content is low, where pores $<5 \mu \mathrm{m}$ represent a proportion of water-filled pores of $7 \%$ (calculated from the water retention curve). We very likely underestimated the diffusion pathways. This limit is not intrinsic to the MOSAIC II model but to its use with microCT images having insufficient resolution regarding the processes studied.

The mineralization of fructose simulated with a simpler approach using the moisture function of RothC also underestimated the experimental mineralization (Fig. 6). The efficiency coefficients were higher for $3 \mathrm{R}, 5 \mathrm{~L}$, and $6 \mathrm{~L}$ and lower for $9 \mathrm{R}$ and $7 \mathrm{R}$ compared to those obtained using the 3-D MOSAIC II approach. The moisture function proposed in the literature, as the one used in RothC, gave similar trends as the one explicitly calculated using our 3-D approach.

\section{Conclusion and perspectives}

The decomposition of soil organic matter is highly impacted by water content, and poor accounting of this control by current soil organic matter dynamics models is a major source of uncertainty (Falloon et al., 2011). The soil water content and energy state has both direct impacts on microbial physiology, especially at extreme values, and indirect impacts via the diffusion of oxygen and that of solutes like soluble carbon and nitrogen compounds (Moyano et al., 2013). These processes affect the microbial decomposition kinetics. Current compartment-based soil organic matter models use empirical functions to describe the effect of limiting water content on decomposition rates (Moyano et al., 2012). In MOSAIC II, the explicit description of the 3-D interactions between decomposition actors and organic matter in an unsaturated habitat reproduced, without using empirical functions, the decrease of decomposition due to the decrease of water content in a realistic way. We simulated explicitly and calibrated for the first time with real data the indirect impact of changing water content on mineralization via the modification of connected water-filled pathways. Our modelling exercise gave results as good as those obtained using a simple moisture function found in the literature. We anticipate that, for more complex 3-D architectures, such as those of soil compared to sand, the simulation using MOSAIC should be superior. Two directions for improvement can be identified: (i) using CT images with a better spatial resolution, as it is increasingly possible using new generation $\mu \mathrm{CTs}$, in order to describe and model the processes within micrometrescale pores; and (ii) describing better the water connectivity at low water content in the model, accounting for water films.
In future studies more scenarios will be tested using more complex systems (real soil and more complex bacterial community) and for different distances between degraders and organic substrates.

Acknowledgements. This work was financed by the French National Agency for Research (ANR SYSCOMM) within the MEPSOM project.

Edited by: R. Conant

\section{References}

Blagodatsky, S. A., Yevdokimov, I. V., Larinova, A. A., and Richter, J.: Microbial growth in soil and nitrogen turnover: model calibration with laboratory data, Soil Biol. Biochem., 30, 1757-1764, 1998.

Chenu, C. and Stotzky, G.: Interactions between microorganisms and soil particles: An overview, in: Interactions between soil particles and microorganisms, edited by: Huang, P. M., Bollag, J. M., and Senesi, N., IUPAC Serie Appl. Geochem., Wiley and Sons, New York, 3-40, 2002.

Coleman, K., Jenkinson, D. S., Crocker, G. J., Grace, P. R., Klir, J., Korschens, M., Poulton, P. R., and Richter, D. D., Simulating trends in soil organic carbon in long-term experiments using RothC-26.3, Geoderma, 81, 29-44, 1997.

Coucheney, E.: Impact of bacterial diversity on community response to climatic factors: a microcosms study on microbial respiration and metabolomics, University of Paris 6 (France), 239 pp., 2009.

Dechesne, A., Owsianiak, M., Bazire, A., Grundmann, G. L., Binning, P. J., and Smets, B. F.: Biodegradation in a partially saturated sand matrix: compounding effects of water content, bacterial spatial distribution, and motility, Environ. Sci. Technol., 44, 2386-2392, 2010.

Dungait, J. A. J., Hopkins, D. W., Gregory, A. S., and Whitmore, A. P.: Soil organic matter turnover is governed by accessibility not recalcitrance, Global Change Biology, 18, 1781-1796, 2012.

Falloon, P., Jones, C. D., Ades, M., and Paul, K.: Direct soil moisture controls of future global soil carbon changes: An important source of uncertainty, Global Biogeochem. Cy., 25, GB3010, 2011.

Garnier, P., Neel, C., Aita, C., Recous, S., Lafolie, F., and Mary, B.: Modelling carbon and nitrogen dynamics in soil with and without straw incorporation, Europ. J. Soil Sci., 54, 555-568, 2003.

Gharasoo, M., Centler, F., Regnier, P., Harms, H., and Thullner, M.: A reactive transport modeling approach to simulate biogeochemical processes in pore structures with pore-scale heterogeneities, Environ. Model. Software, 30, 102-114, 2012.

Gignoux, J., J., H., Hall, D., Masse, D., Nacro, H. B., and Abbadie, L.: Design and test of a generic cohort model of soil organic matter decomposition: the SOMKO model, Global Ecol. Biogeochem., 10, 639-660, 2001.

Gottschalk, P., Bellarby, J., Chenu, C., Foereid, B., Smith, P., Wattenbach, M., Zingore, S., and Smith, J.: Simulation of soil organic carbon response at forest cultivation sequences using C-13 measurements, Organic Geochem., 41, 41-54, 2010. 
Harms, H.: Bacterial growth on distant naphthalene diffusing through water, air, and water-saturated and nonsaturated porous media, Appl. Environ. Microbiol., 62, 2286-2293, 1996.

Holden: Water stress effects on toluene biodegradation by Pseudomonas putida, Biodegradation, 8, 143-151, 1997.

Ingwersen, J., Poll, C., Streck, T., and Kandeler, E.: Micro-scale modelling of carbon turnover driven by microbial succession at a biogeochemical interface, Soil Biol. Biochem., 40, 864-878, 2008.

Jenkinson, D. S. and Coleman, K.: The turnover of organic carbon in subsoils. Part 2. Modelling carbon turnover, Europ. J. Soil Sci., 59, 400-413, 2008.

Kelly, R. H., Parton, W. J., Crocker, G. J., Grace, P. R., Klir, J., Korschens, M., Poulton, P. R., and Richter, D. D.: Simulating trends in soil organic carbon in long-term experiments using the century model, Geoderma, 81, 75-90, 1997.

Kravchenko, A., Falconer, R., Grinev, D., and Otten, W.: Fungal colonization in soils of contrasting managements: modelling fungal growth in 3-D pore volumes of undisturbed soil samples, Ecol. Appl., 21, 1202-1210, 2011.

Long, T. and Or, D.: Dynamics of Microbial Growth and Coexistence on Variably Saturated Rough Surfaces, Microb. Ecol., 58, 262-275, 2009.

Manzoni, S. and Porporato, A.: Soil carbon and nitrogen mineralization: Theory and models across scales, Soil Biol. Biochem., 41, 1355-1379, 2009.

Manzoni, S., Schimel, J. P., and Porporato, A.: Responses of soil microbial communities to water stress: results from a meta-analysis, Ecology, 93, 930-938, 2012

Masse, D., Cambier, C., Brauman, A., Sall, S., Assigbetse, K., and Chotte, J. L.: MIOR: an individual-based model for simulating the spatial patterns of soil organic matter microbial decomposition, Europ. J. Soil Sci., 58, 1127-1135, 2007.

Monga, O., Ngom, N. F., and Delerue, J. F.: Representing geometric structures in 3-D tomography soil images: Application to pore space modelling, Comput. Geosci., 33, 1140-1161, 2007.

Monga, O., Bousso, M., Garnier, P., and Pot, V.: 3-D geometrical structures and biological activity: application to soil organic matter microbial decomposition in pore space, Ecol. Modell., 216, 291-302, 2008.

Monga, O., Bousso, M., Garnier, P., and Pot, V.: Using pore space 3D geometrical modelling to simulate biological activity: Impact of soil structure, Comput. Geosci., 35, 1789-1801, 2009.

Mooney, S. J.: Three-dimensional visualization and quantification of soil macroporosity and water flow patterns using computed tomography, Soil Use Manag., 18, 142-151, 2002.
Moyano, F., Manzoni, S., and Chenu, C.: Responses of soil heterotrophic respiration to moisture availability: An exploration of processes and models (review), Soil Biol. Biochem., 59, 72-85, 2013.

Moyano, F. E., Vasilyeva, N., Bouckaert, L., Cook, F., Craine, J., Curiel Yuste, J., Don, A., Epron, D., Formanek, P., Franzluebbers, A., Ilstedt, U., Kätterer, T., Orchard, V., Reichstein, M., Rey, A., Ruamps, L., Subke, J.-A., Thomsen, I. K., and Chenu, C.: The moisture response of soil heterotrophic respiration: interaction with soil properties, Biogeosciences, 9, 1173-1182, doi:10.5194/bg-9-1173-2012, 2012.

Ngom, N. F., Monga, O., Mohamed, M. M. O., and Garnier, P.: 3-D shape extraction segmentation and representation of soil microstructures using generalized cylinders, Comput. Geosci., 39, 50-63, 2012.

Nunan, N., Wu, K., Young, I. M., Crawford, J. W., and Ritz, K.: Spatial distribution of bacterial communities and their relationships with the micro-architecture of soil, FEMS Microbiol. Ecol., 44, 203 pp., 2003.

Nunan, N., Young, I. M., Crawford, J. W., and Rithz, K.: Bacterial interactions at the microscale - Linking habitat to function in soil, in: The spatial distribution of microbes in the environment, edited by: Franklin, R. B., and Mills, A. L., Kluwer Academic Publishers, New York, 61-86, 2007.

Peth, S., Horn, R., Beckmann, F., Donath, T., Fischer, J., and Smucker, A. J. M.: Three dimensional quantification of intra aggregate pore space features using synchrotron radiation based microtomography, Soil Sci. Soc. Am. J., 72, 897-907, 2008.

Resat, H., Bailey, V., McCue, L. A., and Konopka, A.: Modeling microbial dynamics in heterogeneous environments: growth on soil carbon sources, Microb. Ecol., 63, 883-897, 2012.

Roberson, E. B. and Firestone, M. K.: The relationship between desiccation and exopolysaccharide production by a soil Pseudomonas, Appl. Environ. Microbiol., 58, 1284-1291, 1992.

Sambrook, J., Fritsch E. F., and Maniatis T.: Molecular cloning: A laboratory manual, 2nd Edn., Cold Spring Harbour Laboratory press, New York, 1989.

Treves, D. S., Xia, B., Zhou, J., and Tiedje, J. M.: A two-species test of the hypothesis that spatial isolation influences microbial diversity in soil, Microb. Ecol., 45, 20-28, 2003.

Wildenschild, D. and Sheppard, A. P.: X-ray imaging and analysis techniques for quantifying pore-scale structure and processes in subsurface porous medium systems, Adv. Water Resour., 51, 217-246, 2013.

Young, I. M., Crawford, J. W., Nunan, N., Otten, W., and Spiers, A.: Microbial distribution in soils: physics and scaling, Adv. Agronomy, 100, 80-121, 2008. 